\title{
BMJ Open Diet quality, food insecurity and risk of cardiovascular diseases among adults living with HIV/AIDS: a scoping review protocol
}

\author{
Iyanuoluwa Oyedeji Oyetunji (D) , ${ }^{1}$ Alastair Duncan (D) , ${ }^{2}$ Sharmilah Booley, ${ }^{3}$ \\ Janetta Harbron (i) ${ }^{1}$
}

To cite: Oyetunji 10,

Duncan A, Booley S, et al. Diet quality, food insecurity and risk of cardiovascular diseases among adults living with HIV/AIDS: a scoping review protocol. BMJ Open 2021;11:e047314. doi:10.1136/ bmjopen-2020-047314

- Prepublication history for this paper is available online. To view these files, please visit the journal online (http://dx.doi. org/10.1136/bmjopen-2020047314).

\section{SB deceased}

Received 24 November 2020 Accepted 24 September 2021

Check for updates

(C) Author(s) (or their employer(s)) 2021. Re-use permitted under CC BY-NC. No commercial re-use. See rights and permissions. Published by BMJ.

${ }^{1}$ Research Centre for Health through Physical Activity, Lifestyle \& Sport, Division of Physiological Sciences, Department of Human Biology, Faculty of Health Sciences, University of Cape Town, Cape Town, South Africa

${ }^{2}$ Department of Nutrition and Dietetics, King's College London, London, UK

${ }^{3}$ Division of Physiological

Sciences, Department of Human Biology, University of Cape Town, Cape Town, South Africa

Correspondence to lyanuoluwa Oyedeji Oyetunji; iyanujesu95@gmail.com

\section{ABSTRACT}

Introduction Cardiovascular diseases (CVDs) are the single greatest contributor to global mortality. The successful introduction and scale-up of antiretroviral therapy (ART) delivered a reduction in HIV mortality. Consequently, an association was found between the scale-up of ART and an increased prevalence of comorbidities among people living with HIV (PLWH) such as hypertension and dyslipidaemia. A higher quality diet can delay the onset of comorbidities related to HIV infection. Diet quality and its methods of assessment are not fully established among PLWH. This review will identify the diet quality and food insecurity indices that have been used among PLWH and how these constructs are associated with risk of developing CVD.

Methods and analysis The frameworks recommended by Arksey and 0'Malley and the Joanna Briggs Institute's manual for conducting scoping reviews will be adopted. The Preferred Reporting Items for Systematic Reviews and Meta-Analyses extension for Scoping Reviews guidelines will be used for reporting. A search strategy was developed using keywords related to the topic. A preliminary MEDLINE (via PubMed) search was conducted on 11 November 2020 to develop a comprehensive search strategy. The final search will be conducted on PubMed, EbscoHost, Scopus, Web of Science and Cochrane Library databases. Titles and abstracts of retrieved records will be screened independently by two reviewers. Data will be extracted from records that meet the inclusion criteria using a predesigned charting tool. Discrepancies in decisions made by reviewers will be resolved by consensus or the decision of a third reviewer. Extracted data will be presented in tables or charts. A descriptive summary of the charts or tables will follow.

Ethics and dissemination Ethical approval is not required for a scoping review. Findings will inform other studies currently underway and will be presented at conferences and published in peer-reviewed journals. Registration number https://osf.io/7k3ja.

\section{INTRODUCTION}

Globally, cardiovascular diseases (CVDs) are the leading cause of death, ${ }^{12}$ with hypertension, diabetes and dyslipidaemia identified as principal risk factors for the development of
Strengths and limitations of this study

- This will be the first scoping review to explore the diet quality and food security status of people living with HIV with or at risk of cardiovascular disease.

- The Preferred Reporting Items for Systematic Reviews and Meta-Analyses extension for Scoping Reviews will be used; this will ensure transparent reporting of findings.

- The scoping review will be based on a comprehensive search strategy that was designed in collaboration with a research librarian and includes sources from seven databases and grey literature.

- A major limitation of our review is the inclusion of only studies published in English language.

- Only published articles in peer-reviewed journals and databases of grey literature will be included.

CVD. ${ }^{3}$ In people living with HIV (PLWH), a threefold increase in the global burden of HIVrelated CVD has been reported over the last two decades. A systematic review concluded that PLWH are twice as likely to develop CVD compared with their HIV-negative counterparts. ${ }^{4}$ This finding could be explained by several contributing factors including the infection itself and its treatment.

It is known that the successful introduction and scale-up of effective highly active antiretroviral therapy (HAART) brought about a reduction in HIV mortality rates, and an ageing cohort of PLWH. ${ }^{5}$ This, however, has been followed by a higher risk of morbidity and increased prevalence of comorbidities including obesity, hyperglycaemia dyslipidaemia, hypertension and other CVDs among PLWH. ${ }^{6} 7$ Furthermore, certain CVD risk factors such as lipodystrophy, increased central adiposity, insulin resistance and diabetes have also directly been linked with the use of HAART. ${ }^{8}$ HIV infection and HAART use have, therefore, 
been reported to significantly increase the risk of CVD. ${ }^{1011}$ Cardiovascular risks are also affected by other lifestyle factors such as dietary intake, smoking and physical activity. ${ }^{12}$

Similarly to the general population, lifestyle modification is an essential first step in the management of CVD among PLWH. Dietary interventions have been demonstrated to reduce the risk of CVD among the HIVuninfected population ${ }^{13-16}$ and PLWH. ${ }^{14}{ }^{15}$ However, there is an absence of HIV-specific dietary recommendations for the reduction of CVD risks among PLWH. ${ }^{17}$

\section{Diet quality}

Diet quality is a concept that is not clearly defined; no consensus has been reached to have a specific meaning that can be applied in all contexts. ${ }^{18}$ Diet quality indices are generally developed to reflect how much an individual or population's food consumption conforms to dietary guidelines and recommendations within a context. ${ }^{18}$ Diet quality is being increasingly adopted in nutritional epidemiology surveys to assess dietary patterns and evaluate the effectiveness of a specific dietary intervention. Since a relationship has been established and understood between food and human physiological function, diet quality has also been used as a proxy to predict mortality and risk of chronic diseases. ${ }^{19} 20$

Diet quality has been measured in diverse ways. Some studies have assessed and compared the intake of a specific nutrient or food components with recommended dietary standards or guidelines. ${ }^{21} 22$ However, it has been argued that overall dietary pattern or the consumption of food groups is a better indication of diet quality compared with the intake of a single nutrient. ${ }^{23}{ }^{24}$ Diet quality indices have been, therefore, designed as a tool to connect food and nutrient intake to the incidence of chronic diseases, mortality and morbidity. ${ }^{23}$ Most epidemiological studies have, since then, measured diet quality using scientifically robust indices enabling standardised assessment. ${ }^{182526}$

\section{Diet quality indices}

Several diet quality indices have been developed and used over the years. Some have been used to evaluate adherence to dietary guidelines while others monitor changes in dietary patterns over time. ${ }^{26}$ Diet quality indices have also been used to identify unfavourable patterns of intake. ${ }^{27}$ Components assessed in diet quality indices include intake of specific macronutrients or micronutrients, adherence to recommended serving sizes of food groups or inclusion of predefined healthy food items. ${ }^{187}$ In summary, diet quality has been used to measure both inclusion of specific foods and nutrients, and variety of diet.

Examples of diet quality indices include:

- Healthy Eating Index (HEI) which was designed based on the Dietary Guidelines for Americans and other dietary patterns set by the US Department of Agriculture (USDA). ${ }^{28-30}$
- Mediterranean Diet Score assessing degree of adherence to Mediterranean dietary guidelines among adults including the elderly. ${ }^{31}$

- Diet Quality Index (DQI) designed to reflect risk of common diet-related diseases,${ }^{24}$ further updated and renamed as DQI-International. ${ }^{32}$

- Recommended Food Score which contains 23 food items and measures overall food quality. ${ }^{33}$

- Dietary Diversity Score ${ }^{34}$ and Food Variety Score, ${ }^{35}$ which are the total count of food groups and food items consumed, respectively, by a unit of population (household or individual) over a specified period of time. This does not put into account the quantity of food or food groups.

- Dietary Approaches to Stop Hypertension diet score which is based on eight food and nutrient components and high in fruits and vegetables. ${ }^{36}$

- Dietary Inflammatory Index which predicts level of inflammatory markers and their outcome on health. ${ }^{37}$

Due to the complex and dynamic nature of diet quality, several reviews investigating associations between diet quality indices and disease risks have been conducted in the general population. ${ }^{25-27} 38-40$ Poor diet quality increases the risk of mortality and morbidity in the HIVuninfected population. ${ }^{41}$ Some studies have also evaluated diet quality among PLWH. ${ }^{62-48}$ Researchers from Boston in the USA conducted a cross-sectional study using the HEI tool, and reported that diet quality was lower among PLWH and significantly lower among women living with HIV when compared with HIV-negative controls. ${ }^{6}$ This study did not link results with risk of CVD.

\section{Food insecurity}

Food insecurity is defined as limited availability of and access to sufficient, safe and nutritious food to support healthy living. ${ }^{49}{ }^{50}$ The Food and Agriculture Organization (FAO), in the most recent report on the state of global food security and nutrition, estimated that 690 million people are hungry, equivalent to $8.9 \%$ of the world population. The FAO projects that the COVID-19 pandemic will exacerbate global food insecurity through disrupting social and economic systems, potentially resulting in up to additional 132 million people experiencing undernutrition in $2020 .^{51}$

Socioeconomic factors such as food insecurity can influence diet quality. Muhammad et a $\tilde{l}^{2}$ reported that $55 \%$ of their sample of PLWH in the USA (aged 50 years and older) are food insecure, and that food insecurity was linked to lower diet quality, irrespective of income. ${ }^{52}$ This finding is supported by evidence in the general population $^{53}$ and corroborated by the FAO report. ${ }^{43}$ Given the current food security situation and the link with diet quality, we will include studies that assess food security status in our review.

\section{Measures of food security}

Food security has been assessed by several indicators at national, household and individual levels. Some 
indicators measure food consumption adequacy while others gather additional information on experiences and behavioural responses. ${ }^{54}$ There have been several paradigms in the concept of food security which have influenced the formulation of new indices. Focus has shifted from global and national food security measures alone to include additional household and individual measures. ${ }^{55}$

Food security indicators may include:

- Food Consumption Score which is used to assess food security and vulnerability by the World Food Program. ${ }^{56}$

- Household Dietary Diversity Score which is seen as the simplest possible measure at the household level. ${ }^{57}$

- Household Food Security Survey Module developed by the USDA. ${ }^{58}$

- Household Food Insecurity Access Scale, ${ }^{49}$ used by the Food and Nutrition Technical Assistance-II initiative. ${ }^{54}$

- Food Insecurity Experience Scale developed by FAO. ${ }^{59}$

The extent to which diet quality and food security status has been assessed in the context of HIV is not known. This scoping review is necessary to aggregate information on the depth of research on diet quality and HIV.

\section{Aims}

The aims of this review include the following:

- To determine the diet quality and food security status of PLWH with or at risk of CVD.

- To identify the range and utility of diet quality and food security indices among PLWH with or at risk of CVD.

\section{METHODS AND ANALYSIS}

The use of scoping reviews to synthesise evidence has increased over the years. As with other forms of literature reviews, they serve general functions of collection, evaluation and presentation of available research evidence. ${ }^{60}$ Scoping reviews can also be termed 'scoping studies' and 'mapping reviews'. ${ }^{61} 62$

There are several reasons why conducting a scoping review is appropriate to answer our research aims. The scoping review could be a step leading to a full systematic review. ${ }^{63}$ In this case, it will identify the feasibility of a systematic review and meta-analysis, the availability of sources of evidence and previous systematic reviews that have been conducted. ${ }^{60}$ In line with suggestions made by several authors about the value of scoping reviews, this scoping review will inform us about the current state of knowledge and types of evidence available on our topic of interest, ${ }^{60}$ as well as illuminate knowledge gaps. ${ }^{6364}$ Furthermore, the review will also summarise how research is conducted in the field of interest ${ }^{63}$; appropriate study populations, research designs and tools can be identified. ${ }^{64}$ Finally, key concepts and their definitions will be identified. ${ }^{63}$ These concepts can be classified based on how they relate; their similarities and differences can be identified and yield a 'concept map'. ${ }^{64}$ An example of a recent scoping review explained the concept of formative peer assessment in a healthcare education programme. ${ }^{65}$
Scoping reviews are useful when the field of study is broadly heterogeneous ${ }^{65}$; diet quality has different indices that are broadly used to appraise various components of dietary intake. Similarly, food security has been measured using a variety of indices. This review will specifically provide a summary of the extent to which diet quality and food security have been explored among PLWH while identifying tools that have been used to evaluate these constructs. Given the emerging concerns of risks of CVD among PLWH, we will identify how much of this concept has been explored within the context of diet quality and food security. This could identify grey areas among these concepts of diet quality, food insecurity and risks of CVD among PLWH, and potentially inform areas for future research. All the above reasons justify the use of scoping review methodology for this study. ${ }^{60-64} 66$

The methodology proposed by Arksey and O'Malley ${ }^{60}$ will form the bedrock for this scoping review. Input from Levac et $a b^{67}$ and Peters $e t a l^{68}$ will also be incorporated. The Joanna Briggs Institute (JBI) manual recommends that a protocol stating a stepwise approach to the scoping review be designed and that a set of criteria for including or excluding studies should be determined a priori. These criteria must reflect the aim as well as the questions of the review. ${ }^{69}$

The framework proposed by Arksey and O'Malley consists of six stages of which five are mandatory. The stages are:

1. Identifying a research question.

2. Identifying relevant studies.

3. Study selection.

4. Charting the data.

5. Collating, summarising and reporting the results.

6. Consultation exercise (optional).

Stage 6 will be omitted as this scoping review is not intended to provide evidence to inform clinical decisions. It will however provide an overview of the literature on dietary components related to risks of CVD in PLWH and give an indication whether the type of data is appropriate for meta-analyses. Given the increased risk of CVD in PLWH, this review will also inform how best to assess dietary intake in this cohort.

\section{Stage 1: identifying the review question}

Common to all review methodologies, scoping reviews start with the formulation of a well-defined research topic that helps to clarify the search strategy. ${ }^{70}$ Guidelines recommend a broad approach to develop a scoping review question, enabling generation of the required depth. ${ }^{60} \mathrm{~A}$ review question should identify the population, concept and context (PCC) of the study, as recommended by the $\mathrm{JBI}^{68}$

This review will be carried out to map the breadth of research on diet quality, food security and risk of CVD among PLWH. The primary review question is:

- What is the current diet quality and food insecurity status of PLWH with or at risk of CVD? 
The introduction of HAART in the late $1990 \mathrm{~s}^{71}$ brought a significant change to the health outcomes of PLWH. ${ }^{72}$ The word 'current' has been included as studies published since 1998 will be considered for the purpose of this scoping review. This time period has been selected based on the recorded time for the global scale-up of HAART.

In addition to the main question, this scoping review also seeks to answer the following secondary question:

- What methodologies have been used to assess the dietary quality and food security of PLWH with or at risk of CVD?

\section{Stage 2: identifying relevant studies}

It is recommended that a scoping review should exhaustively include all sources of evidence, published or unpublished, that can provide insights into the research question. ${ }^{60} \mathrm{~A}$ three-stage systematic approach will be adopted for this scoping review. This ensures all peerreviewed published sources of evidence as well as grey literature are captured. ${ }^{68}$ The first stage is a preliminary search of at least two databases to identify and analyse keywords, text words, index terms and Medical Subject Headings (MeSH) terms related to the search. This was completed on MEDLINE (PubMed) and CINAHL (EBSCOhost) as recommended. ${ }^{68}$ During this stage, key search components and other words that relate to them were identified. The MeSH terms obtained from databases will enable linking other terms related to our search components which have not been identified. Abbreviations of key search terms such as "PLWH" and "PLWHIV" were identified during the preliminary search of articles.
Search terms and abbreviations related to various diet quality indices were also identified from published articles.

In the second stage, a full and comprehensive search strategy was developed from the information retrieved and modified to suit each database. The databases that will be searched include PubMed, Africa wide, CINAHL, APA PsycINFO (via EBSCOhost), Scopus, Web of Science, Cochrane Library, and databases for grey literature such as ProQuest and AHRQ Agency for Healthcare Research and Quality. Table 1 contains an example of a preliminary full search strategy for MEDLINE.

The first two steps of the search were conducted with the assistance of a research librarian. The final step is a manual search of the reference list of all identified sources of evidence that meet the inclusion criteria to locate additional studies.

\section{Inclusion criteria}

JBI recommends an agreement between the title, review questions and inclusion criteria, and further points out the PCC guidelines for topic and review questions. JBI recommends that the participants, concepts, context and types of evidence sources be clearly defined a priori and considered when designing the inclusion criteria. ${ }^{68}$ In contrast, Arksey and O'Malley designed their inclusion criteria post hoc using 'type of study, type of intervention care recipient group and caregiver group' based on their review objective. The JBI recommendation is adopted here because stating the inclusion criteria from the start will give clarity to the process of screening articles.

\section{Table 1 Full search strategy for MEDLINE conducted on 11 November 2020}

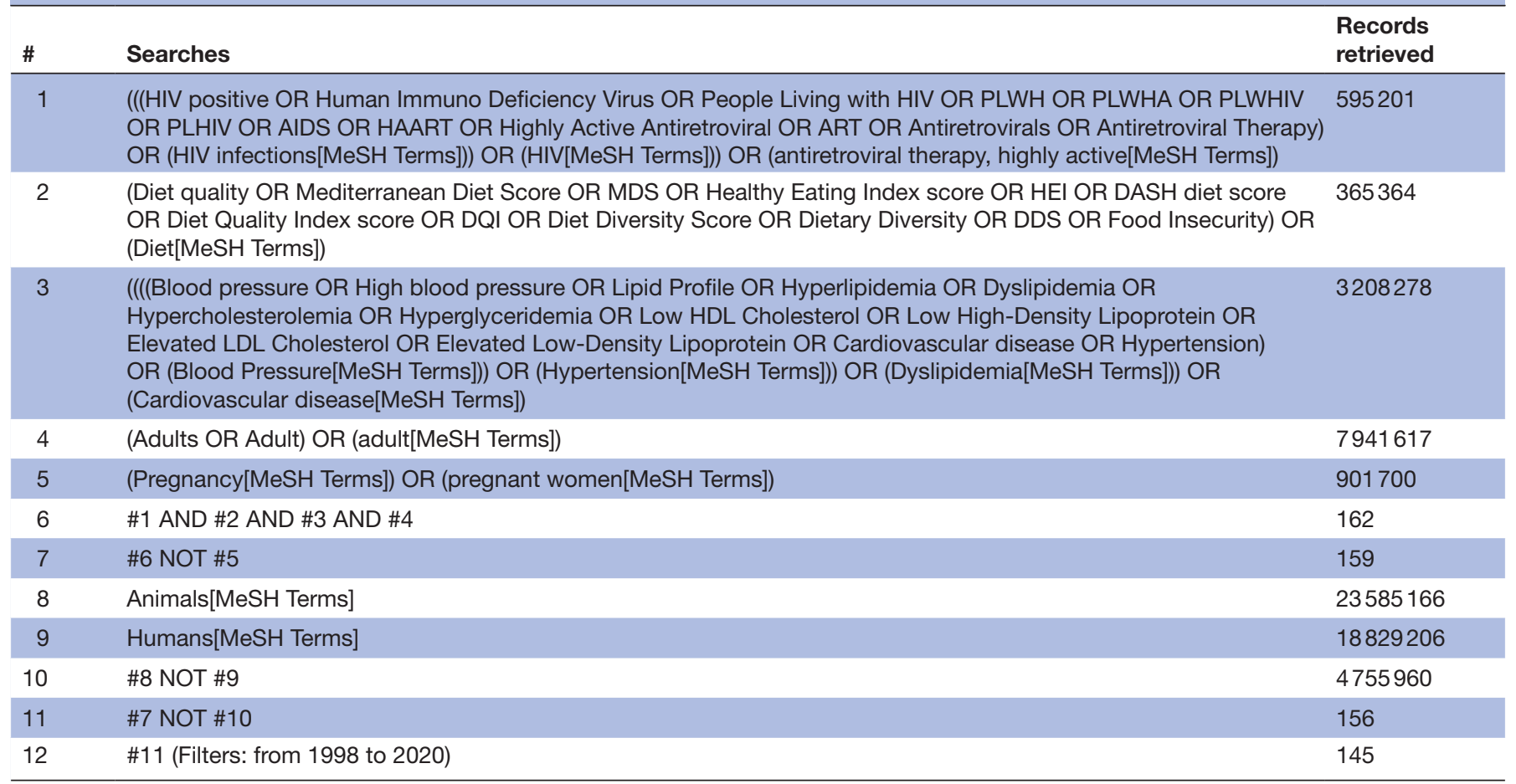

MeSH, Medical Subject Headings. 


\section{Participants}

Studies involving adults living with HIV, non-pregnant, and who are either on HAART or treatment naive will be considered.

\section{Concept}

This review is designed to identify the risk of CVD among adults living with HIV using dietary quality estimates. Dietary quality has been defined above as the degree of adherence to specific dietary guidelines (in this case, guidelines to reduce the risk of CVD). ${ }^{18}$ Therefore, all studies that assess diet quality using either diet quality scores or through the intake of a specific nutrient or food component that relate to the risk of CVD will be included. All diet quality indices will be included in the search strategy. Since it has been established that food insecurity is related to diet quality, ${ }^{52}$ studies that assess food insecurity will also be included.

Food insecurity has been measured using the indices enumerated above. For this review, we will make use of the food insecurity/hunger score or results given by the included sources of evidence.

The risks of CVD that will be considered as the main outcome are hypertension and dyslipidaemia. Since the main exposure we are interested in is diet quality, studies that investigate the risk of CVD without assessing diet quality will not be included.

\section{Context}

Research on dietary recommendations and how they affect health and diseases has expanded over the years. The use of HAART has also changed the narratives of HIV infection. It is therefore ideal to include only recent studies in this review. As stated above, studies published earlier than 1998 will be excluded.

\section{Types of sources}

All primary studies and reviews conducted on human subjects will be included. Laboratory studies, research conducted on biochemical substances and studies that are not published in English will be excluded.

\section{Stage 3: study selection}

After the search has been conducted, the identified and collated citations will be exported into EndNote V.X9 (Clarivate, Analytics, Pennsylvania, USA) to remove duplicates. The new citations will be uploaded to Rayyan QCRI (Copenhagen: The Nordic Cochrane Centre, Cochrane), ${ }^{73}$ where titles and abstracts will be assessed independently against the inclusion criteria by two members of the research team. Disagreements on screened citations will be discussed and resolved by consensus or the intervention of a third reviewer when necessary. This approach is consistent with methodology previously developed and used. ${ }^{606874}$

Full text reports of studies that passed the initial stage of screening will be retrieved and screened to verify their conformance with the inclusion criteria. Articles that fail to meet the inclusion criteria here will be excluded and reasons will be included in the final report.

A full report of the search will be presented in a Preferred Reporting Items for Systematic Reviews and Meta-Analyses extension for Scoping Reviews flow chart and included in the final report. Details of excluded studies and reasons for their exclusion will be compiled from a predefined list, those suggested and agreed on by the reviewers during the process of review will also be included. This list of exclusion criteria will be classified and included in the final report.

\section{Stage 4: charting the data}

Following recommendations, a predesigned tabulated data extraction tool template will be piloted on 10 included studies. ${ }^{68} \mathrm{JBI}$ identified that there usually is a need to chart additional data unforeseen from the time of study design, therefore any modification made to the tool will be detailed in the full report of this review. ${ }^{68}$ This chart helps the reviewers to easily keep track of each source of evidence and gives the reader a quick and logical overview of the results that answer the review questions. $^{60} 73$

Data extracted will be tabulated as follows: first author/ year of publication, country, aim of the study, population/sample size, study design, participant recruitment, duration of study, diet quality index used and/or food insecurity measure used, outcome, risk of the CVD reported and prevalence, key findings that relate to the review questions, author's conclusion, interpretation and recommendations.

\section{Stage 5: collating, summarising and reporting the results}

Quantitative data extracted will be presented in tables or charts (as appropriate) in line with the review questions. An integrated descriptive summary and interpretation of the charts or tables will follow. Qualitative data will be presented thematically, pre-identified themes that may be reported include types of diet quality index, risk of CVD reported, diet quality status by gender, diet quality status by geographical location. Other themes identified while collating data will also be included. Meta-analysis of data or qualitative evaluation of included studies will not be conducted for this review. This review is intended to give a descriptive overview of diet quality, food insecurity status and risk of CVD of adults living with HIV.

\section{Stage 6: consultation}

This stage is optional and is not planned to be conducted for this scoping review.

\section{Patient and public involvement}

No patient involved.

\section{ETHICS AND DISSEMINATION}

The review will not require any generation of primary data; all documents will be retrieved from the public domain. This review, therefore, does not require ethical 
approval. It forms part of dissertation towards a Master of Medical Science in Nutrition which is underway. Results will be presented at conferences and published in a peerreviewed journal. This protocol is registered on Open Science Framework with registration number: https:// osf.io/7k3ja.

\section{Twitter lyanuoluwa Oyedeji Oyetunji @lyanu_Jesu}

Acknowledgements The authors acknowledge and appreciate the effort of Namhla Madini of the Bongani Mayosi Health Sciences Library, the University of Cape Town for the assistance she provided with the preliminary search and developing the search strategy.

Contributors All authors were involved in the conceptualisation of the scoping review protocol. 100 led the process, drafted the protocol and wrote the manuscript under the supervision of $\mathrm{JH}, \mathrm{SB}$ and $\mathrm{AD}$. All authors approved the publishing of this protocol.

Funding The Mastercard Foundation Scholars Program funds the degree of I00.

Disclaimer This work does not reflect the views of the funder, but the views of the authors based on research findings.

Competing interests None declared.

Patient and public involvement Patients and/or the public were not involved in the design, or conduct, or reporting, or dissemination plans of this research.

Patient consent for publication Not required.

Provenance and peer review Not commissioned; externally peer reviewed.

Open access This is an open access article distributed in accordance with the Creative Commons Attribution Non Commercial (CC BY-NC 4.0) license, which permits others to distribute, remix, adapt, build upon this work non-commercially, and license their derivative works on different terms, provided the original work is properly cited, appropriate credit is given, any changes made indicated, and the use is non-commercial. See: http://creativecommons.org/licenses/by-nc/4.0/.

\section{ORCID iDs}

Iyanuoluwa Oyedeji Oyetunji http://orcid.org/0000-0001-7602-1463

Alastair Duncan http://orcid.org/0000-0002-6284-8192

Janetta Harbron http://orcid.org/0000-0001-9273-1190

\section{REFERENCES}

1 World Health Organization. Hypertension, 2019. Available: https:// www.who.int/news-room/fact-sheets/detail/hypertension

2 Naghavi M, Abajobir AA, Abbafati C, et al. Global, regional, and national age-sex specific mortality for 264 causes of death, 19802016: a systematic analysis for the global burden of disease study 2016. Lancet 2017;390:1151-210.

3 World Health Organization. Cardiovascular diseases, 2017. Available: https://www.who.int/news-room/fact-sheets/detail/cardiovasculardiseases-(cvds)

4 Shah ASV, Stelzle D, Lee KK, et al. Global burden of atherosclerotic cardiovascular disease in people living with HIV: systematic review and meta-analysis. Circulation 2018;138:1100-12.

5 Mutevedzi PC, Newell M-L. The changing face of the HIV epidemic in sub-Saharan Africa. Trop Med Int Health 2014;19:1015-28.

6 Weiss JJ, Sanchez L, Hubbard J, et al. Diet quality is low and differs by sex in people with HIV. J Nutr 2019;149:78-87.

7 Rasmussen LD, May MT, Kronborg G, et al. Time trends for risk of severe age-related diseases in individuals with and without HIV infection in Denmark: a nationwide population-based cohort study. Lancet HIV 2015;2:e288-98.

8 Bozkurt B. Cardiovascular toxicity with highly active antiretroviral therapy: review of clinical studies. Cardiovasc Toxicol 2004;4:243-60.

9 Rawdanowicz J, Pikto-Pietkiewicz W, Marczyńska M. Cardiovascular diseases associated with HIV infection and their management. Kardiol Pol 2013;71:1183-7.

10 Nou E, Lo J, Grinspoon SK. Inflammation, immune activation, and cardiovascular disease in HIV. AIDS 2016;30:1495-509.

11 Nduka C, Sarki A, Uthman O, et al. Impact of antiretroviral therapy on serum lipoprotein levels and dyslipidemias: a systematic review and meta-analysis. Int J Cardiol 2015;199:307-18.
12 Tavares CD, Mesquita AK, Almeida D. Risk factors for cardiovascular diseases in patients with HIV lipodystrophic syndrome. Revista Gestão \& saúde 2012;3:836-51.

13 Estruch R, Ros E, Salas-Salvadó J, et al. Primary prevention of cardiovascular disease with a Mediterranean diet supplemented with extra-virgin olive oil or nuts. N Engl J Med Overseas Ed 2018;378:e34.

14 Lazzaretti RK, Kuhmmer R, Sprinz E, et al. Dietary intervention prevents dyslipidemia associated with highly active antiretroviral therapy in human immunodeficiency virus type 1-infected individuals: a randomized trial. J Am Coll Cardiol 2012;59:979-88.

15 Stradling C, Thomas G, Hemming K. The Mediterranean portfolio diet in HIV dyslipidaemia: a randomized controlled trial. Top Antivir Med 2018;26:306s.

16 Grundy SM, Stone NJ, Bailey AL, et al. 2018 AHA/ACC/AACVPR/ AAPA/ABC/ACPM/ADA/AGS/APhA/ASPC/NLA/PCNA Guideline on the Management of Blood Cholesterol: Executive Summary: A Report of the American College of Cardiology/American Heart Association Task Force on Clinical Practice Guidelines. J Am Coll Cardiol 2019;73:3168-209.

17 Feinstein MJ, Hsue PY, Benjamin LA, et al. Characteristics, prevention, and management of cardiovascular disease in people living with HIV: a scientific statement from the American heart association. Circulation 2019;140:e98-124.

18 Drewnowski A, Henderson SA, Shore AB, et al. Diet quality and dietary diversity in France: implications for the French paradox. $J$ Am Diet Assoc 1996;96:663-9.

19 Alkerwi Ala'a, Aa A. Diet quality concept. Nutrition 2014;30:613-8.

20 Miller V, Webb P, Micha R, et al. Defining diet quality: a synthesis of dietary quality metrics and their validity for the double burden of malnutrition. Lancet Planet Health 2020;4:e352-70.

21 Abioye Al, Isanaka S, Liu E, et al. Gender differences in diet and nutrition among adults initiating antiretroviral therapy in Dar ES Salaam, Tanzania. AIDS Care 2015;27:706-15.

22 Aibana O, Huang C-C, Aboud S, et al. Vitamin D status and risk of incident tuberculosis disease: a nested case-control study, systematic review, and individual-participant data meta-analysis. PLoS Med 2019;16:e1002907.

23 Coulston AM. The search continues for a tool to evaluate dietary quality. Am J Clin Nutr 2001;74:417.

24 Patterson RE, Haines PS, Popkin BM. Diet quality index: capturing a multidimensional behavior. J Am Diet Assoc 1994;94:57-64.

25 Asghari G, Mirmiran P, Yuzbashian E, et al. A systematic review of diet quality indices in relation to obesity. Br J Nutr 2017;117:1055-65.

26 Fransen HP, Ocké MC. Indices of diet quality. Curr Opin Clin Nutr Metab Care 2008;11:559-65.

27 Wirt A, Collins CE. Diet quality--what is it and does it matter? Public Health Nutr 2009;12:2473-92.

28 Reedy J, Lerman JL, Krebs-Smith SM, et al. Evaluation of the healthy eating Index-2015. J Acad Nutr Diet 2018;118:1622-33.

29 Guenther PM, Kirkpatrick SI, Reedy J, et al. The healthy eating Index-2010 is a valid and reliable measure of diet quality according to the 2010 dietary guidelines for Americans. J Nutr 2014;144:399-407.

30 Krebs-Smith SM, Pannucci TE, Subar AF, et al. Update of the healthy eating index: HEl-2015. J Acad Nutr Diet 2018;118:1591-602.

31 Trichopoulou A, Kouris-Blazos A, Wahlqvist ML, et al. Diet and overall survival in elderly people. BMJ 1995;311:1457-60.

32 Kim S, Haines PS, Siega-Riz AM, et al. The diet quality IndexInternational (DQI-I) provides an effective tool for cross-national comparison of diet quality as illustrated by China and the United States. J Nutr 2003;133:3476-84.

33 Kant AK, Schatzkin A, Graubard BI, et al. A prospective study of diet quality and mortality in women. JAMA 2000;283:2109-15.

34 Jayawardena R, Byrne NM, Soares MJ, et al. High dietary diversity is associated with obesity in Sri Lankan adults: an evaluation of three dietary scores. BMC Public Health 2013;13:314.

35 Hatløy A, Torheim LE, Oshaug A. Food variety--a good indicator of nutritional adequacy of the diet? A case study from an urban area in Mali, West Africa. Eur J Clin Nutr 1998;52:891.

36 Fung TT, Chiuve SE, McCullough ML, et al. Adherence to a DASHstyle diet and risk of coronary heart disease and stroke in women. Arch Intern Med 2008;168:713-20.

37 Shivappa N, Steck SE, Hurley TG, et al. A population-based dietary inflammatory index predicts levels of C-reactive protein in the seasonal variation of blood cholesterol study (seasons). Public Health Nutr 2014:17:1825-33.

38 Zaragoza-Martí A, Cabañero-Martínez MJ, Hurtado-Sánchez $\mathrm{JA}$, et al. Evaluation of Mediterranean diet adherence scores: a systematic review. BMJ Open 2018;8:e019033.

39 Schwingshackl L, Bogensberger B, Hoffmann G. Diet quality as assessed by the healthy eating index, alternate healthy eating 
index, dietary approaches to stop hypertension score, and health outcomes: an updated systematic review and meta-analysis of cohort studies. J Acad Nutr Diet 2018;118:74-100.e11.

40 Waijers PMCM, Feskens EJM, Ocké MC. A critical review of predefined diet quality scores. Br J Nutr 2007;97:219-31.

41 Olstad DL, Campbell NRC, Raine KD. Diet quality in Canada: policy solutions for equity. CMAJ 2019;191:E100-2.

42 Duran ACF, Jaime PC. Diet quality of people living with HIV/AIDS: associcated factors. Ann Nutr Metab 2009;55:390.

43 Henderson C. Study results from international food policy research Institute provide new insights into public health (food access and diet quality independently predict nutritional status among people living with HIV in Uganda). AIDS Weekly 2013:a70.

44 Kadiyala S, Rawat R. Food access and diet quality independently predict nutritional status among people living with HIV in Uganda. Public Health Nutr 2013;16:164-70.

45 Palermo T, Rawat R, Weiser SD, et al. Food access and diet quality are associated with quality of life outcomes among HIV-infected individuals in Uganda. PLoS One 2013;8:e62353.

46 Sackey J, Wang G, Wanke C, et al. Socio-Demographic factors associated with diet quality among a cohort of people living with HIV (P04-075-19). Curr Dev Nutr 2019;3.

47 Sackey J, Zhang FF, Rogers B, et al. Food security and dietary diversity are associated with health related quality of life after 6 months of follow up among people living with HIV in Accra, Ghana. AIDS Care 2018;30:1567-71.

48 Stanner H, Radler DR, Sackey J. The association between diet quality and fasting blood glucose in people living with HIV (P08051-19). Curr Dev Nutr 2019;3.

49 Eaton LA, Cain DN, Pitpitan EV, et al. Exploring the relationships among food insecurity, alcohol use, and sexual risk taking among men and women living in South African townships. J Prim Prev 2014;35:255-65.

50 Goosen A, Venter D, Beeforth M, et al. Food insecurity among students living with HIV: strengthening safety nets at the Nelson Mandela metropolitan University, South Africa. Sahara Journal of the Social Aspects of HIVIAIDS 2016;13:106-11.

51 FAO, IFAD, UNICEF, WFP, WHO. The state of food security and nutrition in the world 2020. Rome, Italy: FAO, IFAD, UNICEF, WFP, WHO, 2020: 320 p..

52 Muhammad JN, Fernandez JR, Clay OJ, et al. Associations of food insecurity and psychosocial measures with diet quality in adults aging with HIV. AIDS Care 2019;31:554-62.

53 Hanson KL, Connor LM. Food insecurity and dietary quality in US adults and children: a systematic review. Am J Clin Nutr 2014;100:684-92.

54 Cafiero C, Melgar-Quiñonez HR, Ballard TJ, et al. Validity and reliability of food security measures. Ann N Y Acad Sci 2014;1331:230-48.

55 Maxwell S. Food security: a post-modern perspective. Food Policy 1996;21:155-70.
56 Programme WF. Food consumption analysis: calculation and use of the food consumption score in food security analysis. Italy: World Food Programme Rome, 2008.

57 Swindale A, Bilinsky P. Household dietary diversity score (HDDS) for measurement of household food access: indicator guide. Washington, DC: Food and Nutrition Technical Assistance Project, Academy for Educational Development, 2006.

58 Bickel G, Nord M, Price C, et al. Guide to measuring household food security. revised, 2000.

59 Ahmadi D, Melgar-Quiñonez H. Use of the food insecurity experience scale to assess food security status in Ireland, 2014-17: a crosssectional analysis. The Lancet 2018;392:S16.

60 Arksey H, O'Malley L. Scoping studies: towards a methodological framework. Int J Soc Res Methodol 2005;8:19-32.

61 Anderson S, Allen P, Peckham S, et al. Asking the right questions: Scoping studies in the commissioning of research on the organisation and delivery of health services. Health Res Policy Syst 2008;6:1-12.

62 Ehrich K, Freeman GK, Richards SC. How to do a scoping exercise: continuity of care. Res Policy Plan 2002;20:25-9.

63 Munn Z, Peters MDJ, Stern C, et al. Systematic review or scoping review? guidance for authors when choosing between a systematic or scoping review approach. BMC Med Res Methodol 2018;18:143.

64 Chrastina J. Systematic review and (Aystematic) scoping review: similarities or differences? 2019.

65 Stenberg M, Mangrio E, Bengtsson M, et al. Formative peer assessment in healthcare education programmes: protocol for a scoping review. BMJ Open 2018;8:e025055.

66 Tricco AC, Lillie E, Zarin W, et al. A scoping review on the conduct and reporting of scoping reviews. BMC Med Res Methodol 2016;16:15

67 Levac D, Colquhoun H, O'Brien KK. Scoping studies: advancing the methodology. Implement Sci 2010;5:69.

68 Peters MD, Godfrey C, Mclnerney P. Chapter 11: scoping reviews. In: Joanna Briggs Institute Reviewer's Manual. The Joanna Briggs Institute, 2017.

69 Oduwole EO, Pienaar ED, Mahomed H, et al. Current tools available for investigating vaccine hesitancy: a scoping review protocol. BMJ Open 2019;9:e033245.

70 Khan KS, Ter Riet G, Glanville J, et al. Undertaking systematic reviews of research on effectiveness: CRD's guidance for carrying out or commissioning reviews. NHS Centre for Reviews and Dissemination, 2001.

71 Pau AK, George JM. Antiretroviral therapy. Infect Dis Clin North Am 2014;:28:371-402.

72 Weldehaweria NB, Abreha EH, Weldu MG, et al. Psychosocial correlates of nutritional status among people living with HIV on antiretroviral therapy: a matched case-control study in central zone of Tigray, Northern Ethiopia. PLoS One 2017;12:e0174082.

73 Ouzzani M, Hammady H, Fedorowicz Z, et al. Rayyan-a web and mobile APP for systematic reviews. Syst Rev 2016;5:210.

74 Armstrong R, Hall BJ, Doyle J, et al. 'Scoping the scope' of a cochrane review. J Public Health 2011;33:147-50. 